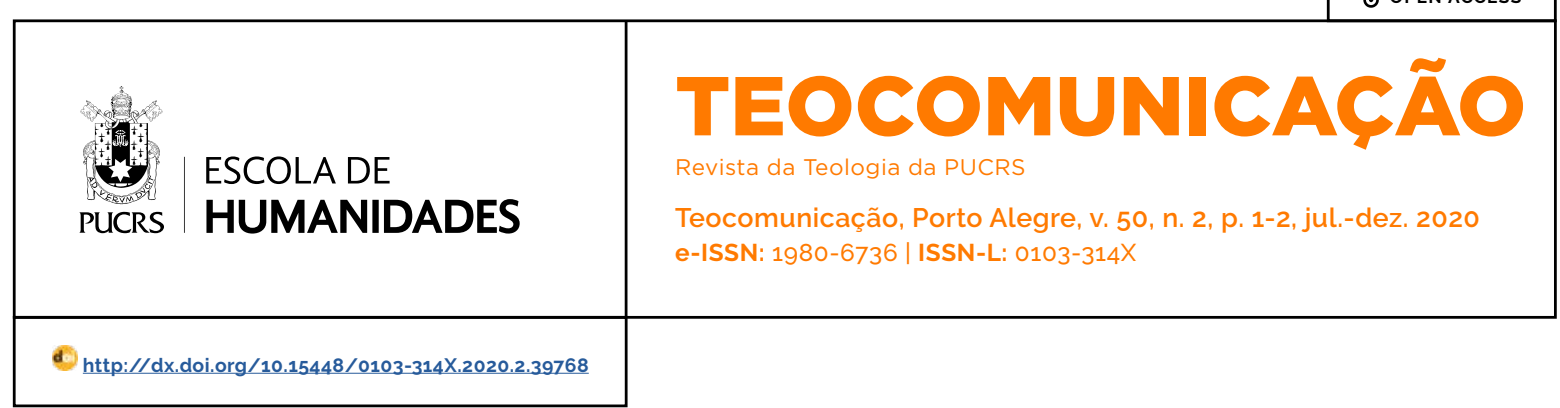

\title{
NOMINATA DOS AVALIADORES DA TEOCOMUNICAÇÃO 2020
}

\author{
LIST OF REVIEWERS - TEOCOMUNICAÇÃO 2020
}

A equipe editorial agradece a contribuição dos pareceristas que avaliaram os artigos e resenhas da Revista Teocomunicação, ano 2020:

Adriano Lima - Faculdade Batista do Paraná - PR - Brasil

Agemir Bavaresco - Pontifícia Universidade Católica do Rio Grande do Sul - Porto Alegre - RS - Brasil

Angela Maringoli - Universidade Metodista de São Paulo - São Bernardo do Campo - SP - Brasil

Antonio Francisco Jacaúna - Pontifícia Universidade Católica de Uberlândia - MG - Brasil

Boris Augustin Nef Ulloa - Pontificia Universidade Católica de São Paulo - São Paulo - SP - Brasil

Bruno Odelio Birck - Pontificia Universidade Católica do Rio Grande do Sul - Porto Alegre - RS - Brasil

Cássio Murilo Dias da Silva - Pontificia Universidade Católica do Rio Grande do Sul - Porto Alegre - RS - Brasil

Claudio Vicente Immig - Pontifícia Universidade Católica do Rio Grande do Sul - Porto Alegre - RS - Brasil

Clélia Peretti - Pontifícia Universidade Católica do Paraná - PR - Brasil Clodomiro de Sousa e Silva - Instituto Católico de Estudos Superiores do Piaui - Teresina - Piaui - PI - Brasil

Edson de Faria Francisco - Universidade Metodista de São Paulo - São Bernardo do Campo - SP - Brasil

Erico João Hammes - Pontificia Universidade Católica do Rio Grande do Sul - Porto Alegre - RS - Brasil

Everaldo Cescon - Universidade de Caxias do Sul - RS - Brasil

Fabrizio Zandonadi Catenassi - Pontificia Universidade Católica do Paraná - Curitiba - PR - Brasil

Fernando César Chaves Reis - Faculdade Católica de Fortaleza - Fortaleza - CE - Brasil 
Flávio Martinez de Oliveira - Universidade Católica de Pelotas - Pelotas - RS - Brasil

Flávio Schmitt: Escola Superior de Teologia - São Leopoldo - RS - Brasil

Geraldo Luiz Borges Hackmann - Pontifícia Universidade Católica do Rio Grande do Sul - Porto Alegre - RS - Brasil

Gilvan Leite de Araujo - Pontifícia Universidade Católica de São Paulo - São Paulo - SP - Brasil

Gustavo Haas - Pontifícia Universidade Católica do Rio Grande do Sul - Porto Alegre - RS - Brasil Ildo Perondi - Pontificia Universidade Católica do Paraná - Curitiba - PR - Brasil

Isidoro Mazzarolo - Pontificia Universidade Católica do Rio Grande do Sul - Porto Alegre - RS - Brasil Jean Richard Lopes - Pontifícia Universidade Católica de Minas Gerais - Belo Horizonte - MG - Brasil João Leonel - Universidade Presbiteriana Mackenzie - São Paulo - SP - Brasil.

José Gabriel Perissé Madureira - Pontificia Universidade Católica do Rio Grande do Sul - Porto Alegre - RS - Brasil

Klaus Da Silva Raupp - Boston College - Boston - USA

Leandro Luis Bedin Fontana - Philosophisch-Theologische Hochschule Sankt Georgen - Frankfurt - Alemanha

Leandro Luis Bedin Fontana - Goethe Universität Frankfurt am Main - Frankfurt - Alemanha.

Ludinei Marcos Vian - Pontificia Universidade Católica do Rio Grande do Sul - Porto Alegre - RS - Brasil Lygia Maria Bitencourt Moura Oliveira - Universidade de Brasilia - Brasilia - DF - Brasil

Marcelo da Silva Carneiro: Faculdade da Igreja Presbiteriana Independente - São Paulo - SP - Brasil Matthias Grenzer: Pontificia Universidade Católica de São Paulo - São Paulo - SP - Brasil

Paulo Sérgio de Proença: Universidade da Integração Internacional da Lusofonia Afro-Brasileira - São Francisco do Conde - BA - Brasil
Rafael Martins Fernandes - Pontifícia Universidade Católica do Rio Grande do Sul - Porto Alegre - RS - Brasil

Romano Dellazari - Pontificia Universidade Católica do Rio Grande do Sul - Porto Alegre - RS - Brasil Sérgio Junqueira Junqueira - Instituto de Pesquisa e Formação de Educação e Religião - Curitiba (PR) - Brasil

Talis Pagot - Pontifícia Universidade Católica do Rio Grande do Sul - Porto Alegre - RS - Brasil

Tiago De Fraga Gomes - Pontificia Universidade Católica do Rio Grande do Sul - Porto Alegre RS - Brasil

Valmor da Silva: Pontificia Universidade Católica de Goiás - Goiânia - GO - Brasil

Valtair Afonso Miranda: Universidade Federal do Rio de Janeiro - Rio de Janeiro - RJ - Brasil

Vicente Artuso: Pontifícia Universidade Católica do Paraná - Curitiba - PR - Brasil

William Lacy Lane: Universidade Presbiteriana Mackenzie - Campinas - SP - Brasil

Wilmar Luiz Barth - Pontificia Universidade Católica do Rio Grande do Sul - Porto Alegre - RS - Brasil 